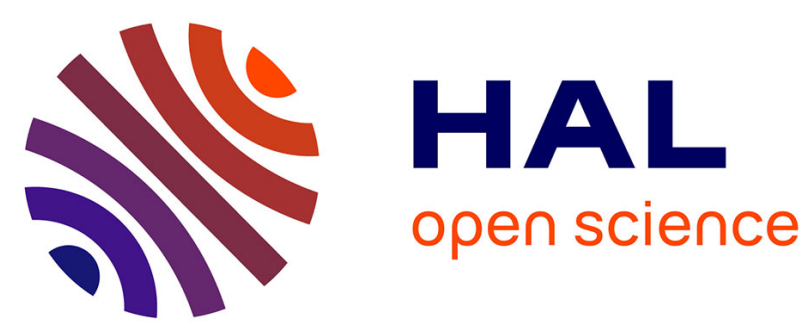

\title{
Underdetermined Source Separation of Finite Alphabet Signals Via L1 Minimization
}

\author{
Si Mohamed Aziz Sbai, Abdeldjalil Aissa El Bey, Dominique Pastor
}

\section{To cite this version:}

Si Mohamed Aziz Sbai, Abdeldjalil Aissa El Bey, Dominique Pastor. Underdetermined Source Separation of Finite Alphabet Signals Via L1 Minimization. ISSPA 2012: 11th IEEE International Conference on Information Sciences, Signal Processing and their Applications, Jul 2012, Montreal, Quebec, Canada. pp.625 - 628. hal-00725038v2

\section{HAL Id: hal-00725038 \\ https://hal.science/hal-00725038v2}

Submitted on 20 Aug 2015

HAL is a multi-disciplinary open access archive for the deposit and dissemination of scientific research documents, whether they are published or not. The documents may come from teaching and research institutions in France or abroad, or from public or private research centers.
L'archive ouverte pluridisciplinaire HAL, est destinée au dépôt et à la diffusion de documents scientifiques de niveau recherche, publiés ou non, émanant des établissements d'enseignement et de recherche français ou étrangers, des laboratoires publics ou privés. 


\title{
UNDERDETERMINED SOURCE SEPARATION OF FINITE ALPHABET SIGNALS VIA L1 MINIMIZATION
}

\author{
Si Mohamed Aziz Sbaï $\quad$ Abdeldjalil Aïssa-El-Bey $\quad$ Dominique Pastor \\ Institut Télécom; Télécom Bretagne; UMR CNRS 3192 Lab-STICC, Technopôle Brest Iroise, France \\ Université européenne de Bretagne, France
}

\begin{abstract}
This paper addresses the underdetermined source separation problem of finite alphabet signals. We present a new framework for recovering finite alphabet signals. We formulate this problem as a recovery of sparse signals from highly incomplete measurements. It is known that sparse solutions can be obtained by $\ell_{1}$ minimization, through convex optimization. This relaxation procedure in our problem fails in recovering sparse solutions. However, this does not impact the reconstruction of the finite alphabet signals. Simulation results are presented to show that this approach provides good recovery properties and good images separation performance.
\end{abstract}

\section{INTRODUCTION}

Source separation problem has been intensively investigated in the literature in the recent three decades. The purpose of source separation systems is to estimate the original source signals from their mixtures. Source separation is an important research topic in a variety of fields, including speech and audio processing [1], radar processing [2], medical imaging [3], and communication [4]. Source separation problems can be classified according to the nature of the mixing process (instantaneous, convolutive) and the ratio between the number of sources and the number of sensors of the problem (determined, underdetermined, overdetermined). This paper is restricted to the noiseless instantaneous case, where each observation consists of a sum of sources with different signal intensity.

Most algorithms solving source separation problem separates the sources in two stages. In the first stage, the unknown mixing matrix is estimated and in the second stage, the sources are estimated. Our contribution focuses on the later stage. We refer readers to [5] for mixing matrix estimation.

Underdetermined source separation (USS) problem, when the number of sources is more than the number of the observed signals, is obviously an ill-posed problem, and its solution cannot be derived without additional assumptions. As an example, in [6], the sources are separated using sparsity of the sources in the time-frequency domain. A source is said to be sparse in a given signal representation domain if most of its samples are zero. Another approach is based on the geometric properties of the signals [7].
This paper deals with separation of finite alphabet signals. Linear combinations of such signals are encountered e.g. in data communications and image processing. In this paper, we show that this problem can be expressed as a sparse recovering problem. Inspired by literature on sparse reconstruction [8], [9], we relax the sparse problem to $\ell_{1}$ minimization. This convex relaxation provides good recovery performance for random sensing matrices when a condition on the number of missing measurements holds.

The layout of this paper is as follows. The next section recalls the data model associated to the source separation problem and describes a basic assumption for identifiability of the original sources. We then propose a new procedure for recovering finite alphabet signals in section 3 . Simulations results are given in section 4 . There is some discussion of the proposed method in section 5. Finally, conclusions are given in section 6 .

\section{PROBLEM STATEMENT}

\subsection{Data model}

Consider the following noise free mixing model:

$$
\boldsymbol{y}=\boldsymbol{\Phi} \boldsymbol{f},
$$

where $\boldsymbol{f}=\left[f_{1}, f_{2}, \cdots, f_{N}\right]^{T}$ is the $N \times 1$ source vector, $\boldsymbol{y}=\left[y_{1}, y_{2}, \cdots, y_{n}\right]^{T}$ is the $n \times 1$ mixture vector and $\boldsymbol{\Phi}$ is the $n \times N$ mixing matrix. Given $\boldsymbol{y}$ and $\boldsymbol{\Phi}$, the purpose of USS is to estimate $\boldsymbol{f}$ in the underdetermined case, i-e when $N>n$. In this case, the system (1) has either no solution or infinitely many solutions. In order to avoid the case of having no solution, we shall suppose that $\boldsymbol{\Phi}$ is a full rank matrix.

The solution of (1) cannot be derived without additional assumptions. In this paper, we consider source signals $\left(f_{i}\right)_{1 \leq i \leq N}$ that only take values from the finite alphabet $\mathcal{A}=\left\{a_{1}, \cdots, a_{p}\right\}$.

\subsection{Solvability assumption}

In order to recover the finite alphabet signal from the underdetermined linear model, it is necessary to assume that:

$\left(\mathcal{H}_{1}\right)$ : Model (1) is $\mathcal{A}$-well-posed. 
in that $\boldsymbol{\Phi} \boldsymbol{f}=\boldsymbol{\Phi} \boldsymbol{f}^{\prime}$ implies $\boldsymbol{f}=\boldsymbol{f}^{\prime}$ [7], where the coefficients of $\boldsymbol{f}$ and $\boldsymbol{f}^{\prime}$ are drawn from the finite alphabet signal $\mathcal{A}$.

It is clear that, without this assumption, it is impossible to identify the original sources with any algorithm. Practically, we note that, for random sensing matrices with reasonable determination, this assumption is easily met.

\section{SEPARATION VIA $\ell_{1}$ MINIMIZATION}

Assuming $\left(\mathcal{H}_{1}\right)$, let $\boldsymbol{f}$ be the unique solution of (1) with coefficients $f_{i} \in \mathcal{A}$. Let $\boldsymbol{\epsilon}_{\boldsymbol{i}}=\left[I\left(f_{i}=a_{1}\right), I\left(f_{i}=\right.\right.$ $\left.\left.a_{2}\right), \cdots, I\left(f_{i}=a_{p}\right)\right]$ denote the indicator vector associated with index $i$, where $I\left(f_{i}=a_{j}\right)$ is the indicator function equal to one when $f_{i}=a_{j}$ and zero otherwise. Put $\hat{\boldsymbol{s}}=\left[\boldsymbol{\epsilon}_{\mathbf{1}}, \boldsymbol{\epsilon}_{\mathbf{2}}, \cdots, \boldsymbol{\epsilon}_{\boldsymbol{N}}\right]^{T} . \hat{\boldsymbol{s}}$ is of length $N p$.

Denote $\boldsymbol{B}_{\boldsymbol{a}}$ and $\boldsymbol{B}_{\mathbf{1}}$ the matrices in $\mathbb{R}^{N \times N p}$ such that:

$$
\begin{aligned}
\boldsymbol{B}_{\boldsymbol{a}} & =\left(\begin{array}{cccc}
\boldsymbol{a} & \mathbf{0}_{p} & \ldots & \mathbf{0}_{p} \\
\mathbf{0}_{p} & \boldsymbol{a} & \ldots & \mathbf{0}_{p} \\
\vdots & \vdots & \vdots & \vdots \\
\mathbf{0}_{p} & \ldots & \mathbf{0}_{p} & \boldsymbol{a}
\end{array}\right) \\
\boldsymbol{B}_{\mathbf{1}} & =\left(\begin{array}{cccc}
\mathbf{1}_{p} & \mathbf{0}_{p} & \ldots & \mathbf{0}_{p} \\
\mathbf{0}_{p} & \mathbf{1}_{p} & \ldots & \mathbf{0}_{p} \\
\vdots & \vdots & \vdots & \vdots \\
\mathbf{0}_{p} & \ldots & \mathbf{0}_{p} & \mathbf{1}_{p}
\end{array}\right)
\end{aligned}
$$

where $\boldsymbol{a}=\left(a_{1}, \cdots, a_{p}\right)$ and $\mathbf{0}_{p}, \mathbf{1}_{p}$ are the row vectors of $\mathbb{R}^{p}$ with respectively zero and one entries only.

Then, it is clear that $\hat{\boldsymbol{s}} \in \mathcal{S}(\boldsymbol{y})$, where $\mathcal{S}(\boldsymbol{y})$ denotes the set $\left\{\boldsymbol{s} \in \mathbb{R}^{N p}: \boldsymbol{\Phi} \boldsymbol{B}_{\boldsymbol{a}} \boldsymbol{s}=\boldsymbol{y}\right.$ and $\left.\boldsymbol{B}_{\mathbf{1}} \boldsymbol{s}=\mathbf{1}_{N}^{T}\right\}$. The first equality results from $\boldsymbol{f}=\boldsymbol{B}_{\boldsymbol{a}} \hat{\boldsymbol{s}}$, the second one is by construction of vector $\hat{\boldsymbol{s}}$.

The following proposition formulates the recovering of finite alphabet signal as a problem of sparse signals recovering from highly incomplete measurements.

Proposition 3.1 Assuming $\left(\mathcal{H}_{1}\right)$, the optimization problem:

$$
\left(P_{0}\right): \quad \arg \min \|s\|_{0} \quad \text { s.t } s \in \mathcal{S}(\boldsymbol{y}) .
$$

where $\|s\|_{0}$ counts the number of nonzero elements in $s$, has a unique solution and is equal to $\hat{s}$.

\section{Proof :}

With assumption $\left(\mathcal{H}_{1}\right)$, let $\boldsymbol{f}$ be the unique solution in $\mathcal{A}^{N}$ of $\boldsymbol{y}=\boldsymbol{\Phi} \boldsymbol{x}$.

On the one hand, we know that $\hat{\boldsymbol{s}} \in \mathcal{S}(\boldsymbol{y})$ and that $\|\hat{\boldsymbol{s}}\|_{0}=$ $N$.

On the other hand, partition the set $\{1,2, \cdots, N p\}$ into $N$ consecutive sets $\left(T_{i}\right)_{1 \leq i \leq N}$ of length $p$ each. Equality $\boldsymbol{B}_{\mathbf{1}} \boldsymbol{s}=\mathbf{1}_{N}^{T}$ implies that the restriction of $s$ to each sets $\left(T_{i}\right)_{1 \leq i \leq N}$ cannot vanish entirely, $\mathrm{i}$-e, each set contains at least one nonzero element of $s$. Thus, for all $s \in \mathcal{S}(\boldsymbol{y})$, $\|s\|_{0} \geq N$.

The unicity of the solution in $x \in \mathcal{A}^{N}$ to $\boldsymbol{y}=\boldsymbol{\Phi} \boldsymbol{x}$ implies that there is no $s \in \mathcal{S}(\boldsymbol{y})$ different from $\hat{\boldsymbol{s}}$ with cardinality $N$.

Thus, $\hat{\boldsymbol{s}}$ is the unique sparse solution in $\mathcal{S}(\boldsymbol{y})$

In order to recover $\boldsymbol{f}$, it is sufficient to find $\hat{s}$ and put $\boldsymbol{f}=\boldsymbol{B}_{\boldsymbol{a}} \hat{\boldsymbol{s}}$. However, finding sparse solutions to underdetermined systems of linear equations is in general NPhard [10]. Solving this problem essentially requires exhaustive search strategy. This method is intractable because the search space is exponentially large. Therefore, inspired by literature on sparse reconstruction [11], we propose to replace the $\ell_{0}$ cost with the $\ell_{1}$ norm to obtain the new recovery procedure of $\boldsymbol{f}$ from $\boldsymbol{y}$. The procedure is described as follows:

- Minimize

$$
\left(P_{1}\right): \quad \arg \min \|\boldsymbol{s}\|_{1} \text { s.t } \boldsymbol{s} \in \mathcal{S}(\boldsymbol{y})
$$

$$
\text { where }\|\boldsymbol{s}\|_{1}=\sum_{i=1}^{N p}\left|s_{i}\right|
$$

- Put $\tilde{\boldsymbol{f}}=\boldsymbol{B}_{\boldsymbol{a}} \tilde{\boldsymbol{s}}$ where $\tilde{\boldsymbol{s}}$ is a solution of $\left(P_{1}\right)$.

On the practical side, unlike the $\ell_{0}$ cost, the $\ell_{1}$-norm is convex. Furthermore, $\left(P_{1}\right)$ problem (for real valued data) can be recast as a linear program. Thus, the new recovery procedure can be solved in a polynomial time. However, problem $\left(P_{1}\right)$ does not always yield the same solution as the $\left(P_{0}\right)$-problem.

The restricted isometry property (RIP) is the most privileged tool to analyze the equivalence between $\left(P_{0}\right)$ and $\left(P_{1}\right)$. The $k$-restricted isometry constant $\delta_{k}$ [12] of a matrix $\mathcal{F}$ is the smallest quantity such that:

$$
\left(1-\delta_{k}\right)\|c\|_{2}^{2} \leq\|\mathcal{F} c\|_{2}^{2} \leq\left(1+\delta_{k}\right)\|c\|_{2}^{2}
$$

holds for all $k$-sparse vectors $c$.

In [13], the author establishes that $\ell_{1}$-problem recovers the solution of $\ell_{0}$-problem provided that $\delta_{2 k}$ of the underdetermined linear system is smaller than 0.4652 . Gaussian random matrices have good restricted isometry constants, in the sense that the RIP holds for large values of $k$. In our case, the RIP condition may not hold. Indeed, even if the mixing matrix $\boldsymbol{\Phi}$ has independent Gaussian entries, the underdetermined system that appears in $\left(P_{0}\right)$ and $\left(P_{1}\right)$

$$
\left(\begin{array}{c}
\Phi B_{a} \\
B_{1}
\end{array}\right)
$$

may no longer satisfy the requirements for equivalence between the two problems. Furthermore, we cannot even guarantee that a solution of $\left(P_{1}\right)$ is unique. However, since we are not interested in recovering the coefficient vector $\hat{\boldsymbol{s}}$, but rather the signal $\boldsymbol{f}=\boldsymbol{B}_{\boldsymbol{a}} \hat{\boldsymbol{s}}$, the equivalence between $\left(P_{0}\right)$ and $\left(P_{0}\right)$ and, thus, the RIP condition, are sufficient but not necessary to estimate the signal. Indeed, the next section shows the performance of our approach for random sensing matrices that do not necessarily verify the RIP condition. 


\section{SIMULATIONS RESULTS}

\subsection{Exact recovery of finite alphabet vector}

In the first set of experiments, pertinence of the approach is experimentally assessed as follows. Let us consider randomly generated signal with $N=256$ samples randomly drawn from a finite alphabet of cardinality $p$. The alphabet can be chosen arbitrarily. Given the number $n$ of measurements, we sample the mixing matrix, for each iteration, with independent Gaussian entries and we compare the recovered $\hat{f}$ and the original $f$ signals.

The recovery is regarded as successful if the relative error $\|\hat{\boldsymbol{f}}-\boldsymbol{f}\|_{2} /\|\boldsymbol{f}\|_{2}$ is less than $10^{-5}$. For each $n$, we repeat 100 iterations of the experiment and average the results. The results are presented in Fig.1 for $p=2$ and $p=4$. Our experiments show that finite alphabet signals can exactly be recovered provided that :

$$
n>\alpha \frac{N(p-1)}{p},
$$

where $\alpha$ controls the probability of success; (e.g. $90 \%$ of success for $\alpha=1.06,100 \%$ of success for $\alpha=1.12$ ). We expect that this result is asymptotic because it involves random matrices and that numerical evaluations show better recovery performance in the limit of large samples.

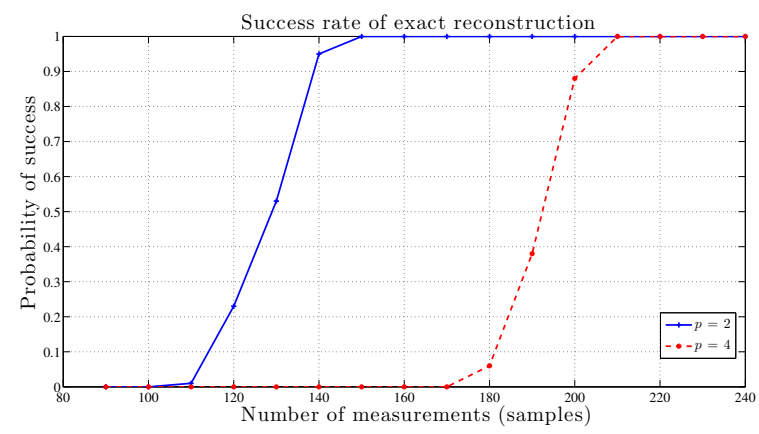

Fig. 1. Success rate of exact reconstruction

When $p=4$, the solution $\tilde{s}$ provided by the standard Cvx routine to problem $\left(P_{1}\right)$ may differ from the solution to problem $\left(P_{0}\right)$. Nevertheless, the estimate $\tilde{\boldsymbol{f}}=\boldsymbol{B}_{a} \tilde{\boldsymbol{s}}$ of $f$ has a relative error less than $10^{-5}$. Therefore, there is no equivalence between $\left(P_{0}\right)$ and $\left(P_{1}\right)$ and, thus, the RIP condition is not satisfied. All the same, this does not impact the good recovery performance of the finite alphabet signals.

\subsection{Binary image separation}

In this experiment, we used four binary images of size $256 \times 256$ (see Fig.2). In order to meet condition (3) of the method, we consider three linear combination of the original images (see Fig.3). The mixing matrix is generated with independent Gaussian entries.

We execute our vectorial procedure by stacking the pixels of the four sources in a one-dimensional signal.

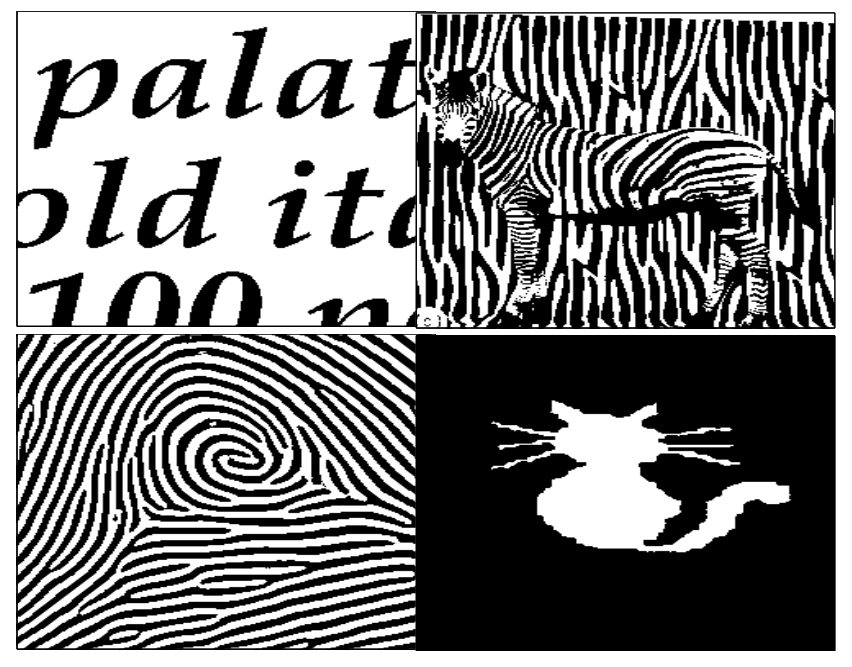

Fig. 2. The Original binary images

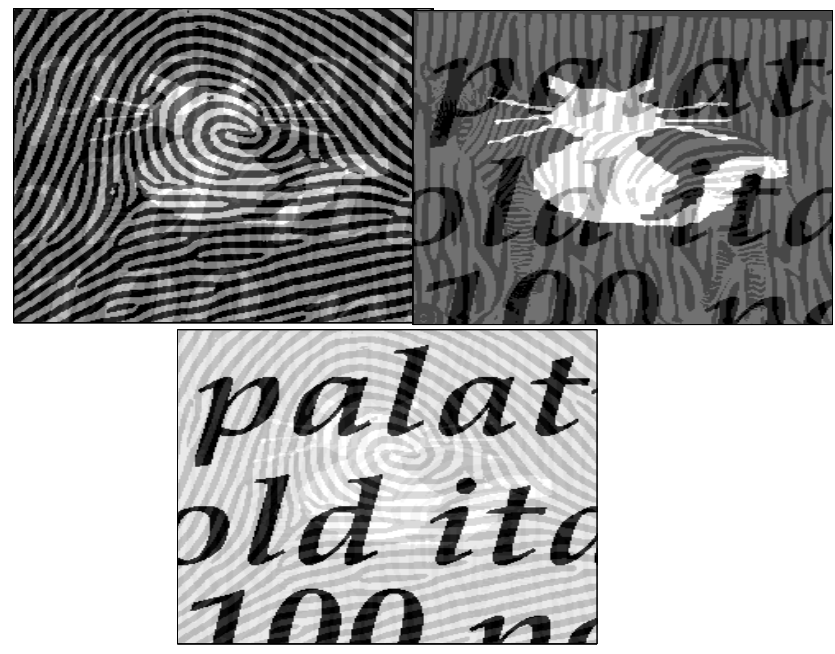

Fig. 3. The observation images

The reconstructed images are shown in Fig.4. We used SNR $=20 \log (\|X\| /\|X-\hat{X}\|)$, where $X$ is the input image, $\|\cdot\|$ stands for the Frobenius norm of a matrix, as performance measure. The following table shows the ability of our method to source separation in images.

\begin{tabular}{|c|c|c|c|c|}
\hline Images & Cat & Text & Zebra & Fingerprint \\
\hline SNR (dB) & 17.15 & 34.73 & 22.49 & 20.23 \\
\hline
\end{tabular}

\section{REMARKS}

- Numerical simulations show that the minimum $\ell_{1}$ norm solution enables recovery of finite alphabet signals for Gaussian matrices when $n>\frac{N(p-1)}{p}$. The proof of this result remains an open issue, which is one of our current research topic.

- The minimization problem in our approach can be related to the $\ell_{1}$-synthesis described briefly in [14]. The authors present novel results concerning the recovery of sparse signals in overcomplete dictionar- 


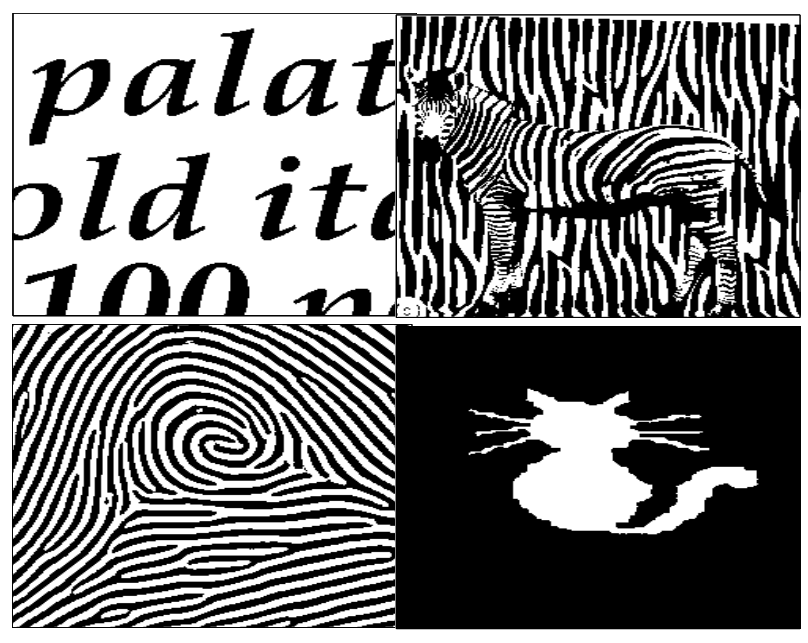

Fig. 4. Separated images using $\ell_{1}$ minimization

ies. The difference with our linear underdetermined source recovery process is that we add observations to our encoding measurements $\left(\boldsymbol{B}_{1} s=\mathbf{1}_{N}^{T}\right)$. Without this step, the recovery algorithm of finite alphabet signals fails.

- The condition $n>\frac{N(p-1)}{p}$ can be rewritten in the form $K p<N$ where $\stackrel{p}{K}$ is the number of missing measurements. Since similar recovery conditions can be found in [15], it can be wondered whether some uncertainty principle would not underly the approach proposed above.

\section{CONCLUSION}

In order to exploit the recent advances in applied harmonic analysis and more precisely the problem of finding the sparsest decomposition of a signal $\boldsymbol{y}$ in a highly overcomplete dictionary $\boldsymbol{\Phi}$, we have presented a new framework for the underdetermined source separation problem of finite alphabet signals. This framework is based on a convex relaxation, which attempts to recover the ideal finite alphabet signal by solving a $\ell_{1}$ optimization. Simulation results illustrate the effectiveness of the proposed approach even when the RIP condition is not satisfied. Therefore, our future investigations would be to establish the theoretical conditions - possibly in connection with heuristic criterion (3) - under which exact reconstruction of finite alphabet signals is possible and to study in details the behavior of our approach in a noisy model.

\section{REFERENCES}

[1] A. Aissa-El-Bey, K. Abed-Meraim, and Y. Grenier, "Underdetermined blind audio source separation using modal decomposition," EURASIP Journal on Audio, Speech, and Music Processing, 2007.

[2] V. Varajarajan and J.L. Krolik, "Multichannel system identification methods for sensor array calibration in uncertain multipath environments," in IEEE Signal Processing Workshop on Statistical Signal Processing (SSP'01), Singapore, 2001, pp. 297-300.

[3] A. Rouxel, D. Le Guennec, and O. Macchi, "Unsupervised adaptive separation of impulse signals applied to EEG analysis," in IEEE International Conference on Acoustics, Speech, Signal Processing, Singapore, 1998, pp. 28882897.

[4] K. Abed-Meraim, S. Attallah, T.J. Lim, and M.O. Damen, "A blind interference canceller in DS-CDMA," in IEEE International Symposium on Spread Spectrum Techniques and Applications, Parsippany, 2000, pp. 358-362.

[5] M. Rostami, M. Babaie-Zadeh, S. Samadi, and C. Jutten, "Blind source separation of discrete finite alphabet sources using a single mixture," in IEEE Statistical Signal Processing Workshop (SSP'11), Nice, France, 2011, pp. 713-716.

[6] S. M. Aziz-Sbai, A. Aissa-El-Bey, and D. Pastor, "Robust underdetermined blind audio source separation of sparse signals in the-frequency domain," in IEEE International Conference on Acoustics, Speech, Signal Processing (ICASSP'11), Prague, Czech Republic, 2011, pp. 37163719.

[7] Y. Li, A. Cichoki, and L. Zhang, "Blind separation and extraction of binary sources," IEICE Transactions on Fundamentals, vol. E86-A, no. 3, pp. 580-589, March 2003.

[8] D. L. Donoho and M. Elad, "Optimally sparse representation in general (nonorthogonal) dictionaries via $\ell_{1}$ minimization," Proc. Nat. Acad. Sci. USA, vol. 100, no. 5, pp. 2197-2202, 2002.

[9] R. Gribonval and M. Nielson, "Sparse representations in unions of bases," IEEE Transactions on Information Theory, vol. 49, no. 12, pp. 3320-3325, December 2003.

[10] B. K. Natarajan, "Sparse approximate solutions to linear systems," ISIAM J. Comput., vol. 24, pp. 227-234, 1995.

[11] D. L. Donoho S. S. Chen and M. A. Saunders, "Atomic decomposition by basis pursuit," SIAM Review, vol. 43, pp. 129-159, 2001.

[12] E. J. Candes and T. Tao, "Decoding by linear programming," IEEE Transactions on Information Theory, vol. 51, no. 12, pp. 4203-4215, December 2005.

[13] S. Foucart, "A note on guaranted sparse recovery via $\ell_{1}$ minimization," App. Comput. Harmon. Anal., vol. 29, no. 1, pp. 97-103, 2010.

[14] D. Needell E. J. Candes, Y. C. Eldar and P. Randall, "Compressed sensing with coherent and redundant dictionaries," In Press, Applied and Computational Harmonic Analysis, 2011.

[15] D. L. Donoho and P. B. Stark, "Uncertainty principles and signal recovery," SIAM J. Appl. Math, vol. 49, pp. 906931, 1989. 